BEATRIZ BATISTA GARCIA

\title{
FORMAÇÃO DOS CONTRATOS ELETRÔNICOS NO DIREITO DO CONSUMIDOR BRASILEIRO
}

\author{
Dissertação de Mestrado
}

Orientador: Professor Associado Dr. Bernardo Bissoto Queiroz de Moraes

Universidade de São Paulo

Faculdade de Direito

São Paulo 
Dissertação apresentado a Banca Examinadora do Programa de Pós-Graduação em Direito, da Faculdade de Direito da Universidade de São Paulo, como exigência parcial para a obtenção do título de Mestre em Direito, na área de concentração Direito Civil, sob a orientação do Professor Associado Dr. Bernardo Bissoto Queiroz de Moraes.

Universidade de São Paulo

Faculdade de Direito

São Paulo 
Garcia, Beatriz Batista

Formação dos contratos eletrônicos no direito do

consumidor brasileiro / Beatriz Batista Garcia ;

orientador Bernardo Bissoto Queiroz de Moraes --'São Paulo, 2017 .

$164 \mathrm{f}$.

Dissertação (Mestrado - Programa de Pós-Graduação em Direito Civil) - Faculdade de Direito, Universidade de São Paulo, 2017.

1. Contratos eletrônicos. 2. Proteção do consumidor. 3. Direito digital. 4. Conclusão contratual. I. Queiroz de Moraes, Bernardo Bissoto, orient. II. Título. 
Nome: GARCIA, Beatriz Batista

Título: Formação dos contratos eletrônicos no direito do consumidor brasileiro

Dissertação apresentada à Faculdade de Direito da Universidade de São Paulo para obtenção do título de Mestre em Direito Civil.

Aprovado em:

BANCA EXAMINADORA

Prof. Instituição:

Julgamento:

Assinatura

Prof.

Instituição:

Julgamento:

Assinatura

Prof.

Instituição:

Julgamento:

Assinatura 
Aos meus pais Laura e Edison, pela paciência e apoio incondicionais e à Julieta, pela alegria proporcionada nessa trajetória. 


\section{AGRADECIMENTOS}

Agradeço à minha avó Maria Luiza (in memoriam), que foi minha segunda mãe e aos meus queridos pais, pela revisão do texto, auxílio em todos os momentos, e, principalmente, pela grande paciência. Sem eles, nada conseguiria.

Gostaria também de prestar eternos agradecimentos ao Professor Bernardo Bissoto Queiroz de Moraes, que me fez descobrir o mundo da pesquisa. Muito obrigada pela compreensão, apoio e ótima orientação.

Merecem homenagem a Faculdade de Direito da Universidade de São Paulo, que considero minha segunda casa; os professores e colegas, principalmente os do grupo de exegese da cadeira de Direito Romano; e os demais funcionários.

Também, agradeço aos amigos e professores da Università degli Studi di Roma "La Sapienza", onde, em 2015, fiquei seis meses aprofundando minha pesquisa e realizei o Corso di Alta Formazione in Diritto Romano.

Agradeço a todos os meus amigos, pelas alegrias, tristezas, dores compartilhas e por sempre compreenderem a minha ausência. Com destaque à verdadeira amizade de Maria Paula de Barros Lopes, Luiza Andrade, Raul Cornélio Silva e Lucas Hiroaki.

Por último, agradeço a todos que de certa maneira participaram e me auxiliaram nessa jornada.

São Paulo, janeiro de 2017. 


\section{Resumo}

GARCIA, B. B.. Formação dos contratos eletrônicos no direito do consumidor brasileiro. 162p. Mestrado - Faculdade de Direito, Universidade de São Paulo, São Paulo, 2016.

Esta dissertação tem por escopo determinar o momento de conclusão dos contratos eletrônicos que envolvam uma relação de consumo, abrangendo aqueles concluídos mediante cliques do consumidor ("click-wrap agreements" ou "point and click") e também mediante a troca de e-mails. Diante das peculiaridades do meio eletrônico, a aplicação das regras tradicionais das etapas de formação contratual, e, principalmente, a precisão do momento da conclusão contratual mostram-se problemáticas.

Em primeiro lugar, foi estudado o momento em que ela ocorre nos contratos entre pessoas presentes e pessoas ausentes no meio material, para depois conferir se o que é aplicado pode também o ser no ciberespaço. Quanto aos contratos não simultâneos entre pessoas ausentes, constatou-se que há a possibilidade de sua formação em quatro momentos, explicados, de modo geral, por quatro teorias: da declaração, da informação, da recepção e da expedição, com destaque às duas últimas, por serem mais usuais.

Os sistemas jurídicos, no entanto, divergem quanto à aplicação de tais teorias, o que torna impossível a sua transposição ao meio eletrônico, já que não possui fronteiras.

Diante do embate entre as teorias da recepção e da expedição, e, apesar de, no Brasil, não haver um consenso, chegou-se ao entendimento de que o melhor momento para a conclusão do contrato eletrônico seria o da recepção da aceitação pelo proponente. Dentre outros motivos, ressalta-se que a teoria da recepção provavelmente é a que mais equilibra os riscos entre as partes.

No entanto, apesar de definido tal momento, para melhor precisá-lo foram cotejadas certas peculiaridades do meio eletrônico, passíveis de discussões se são capazes ou não de influenciá-lo, como, por exemplo: o envio, pelo proponente, de um aviso de recebimento da aceitação; a obrigatoriedade do direito de arrependimento do consumidor; a reserva no "carrinho de compras" pelo consumidor; o aguardo do fornecedor pela aprovação do pagamento. Concluiu-se, ao final, que nenhum desses elementos influencia o momento de conclusão do contrato eletrônico.

Palavras-chave: conclusão do contrato eletrônico, click-wrap agreement, proteção do consumidor, teoria da recepção, teoria da expedição, mailbox rule. 


\section{Abstract}

GARCIA, B. B.. The conclusion of electronic contracts in the brazilian consumer law. 162p. Master - Faculty of Law, University of São Paulo, São Paulo, 2016.

This dissertation intends to define the exact moment of electronic consumer contracts conclusion, specifically those that are concluded by the consumer's clicks (called as "click-wrap agreements" or "point and click" contracts) and also by the exchange of e-mails. Due to electronic environment particularities, the use of traditional rules of the contract formation, and, mainly, the establishment of the moment of the contract conclusion, have become problematic.

First of all, it has been studied the moment of conclusion in the material world, between present and absent people, with the purpose of, then, verify whether the rule usually applied is also suitable for the cyberspace. Regarding the non-simultaneous contracts between absent people, it was possible to verify its formation in four moments, explained, in general, by four theories: declaration, information, receipt and mailbox rules, with emphasis being placed on the last two, because of their prevalent usage.

The legal systems, however, disagree about the application of those theories, becoming impossible to transpose them to cyberspace, since it has no boundaries.

In face of the clash between the receipt and the dispatch rules, and, in spite of the fact that does not exist consensus in Brazil, an understanding has been reached: the best moment for concluding the electronic contracts occurs when there is the receipt of the acceptance by the offeror. Among other reasons, the receipt theory might be the most suitable to the cyberspace because is the one that brings more balance to both parties.

However, notwithstanding the endorsement of the reception theory, to better precise its application, some peculiarities had been analysed in order to discover if they are capable of influenciate the contractual formation: the dispatch of a receiving note by the offeror; the compulsory consumer right of withdrawal; the consumer reservation of the product in the "shopping basket"; and the suppliers waiting of the payment approval. In the end, it has been concluded that none of these listed elements influenciate the moment of the electronic contract conclusion.

Keywords: electronic contract conclusion, click-wrap agreements, consumer protection, receipt rule, dispatch rule, mailbox rule 


\section{Principais abreviaturas}

$\mathrm{CC}$

CDC

1990)

Contratos B2B

Contratos B2C

Contratos B2PA

Contratos C2PA

Contratos $\mathrm{C} 2 \mathrm{C}$

Contratos P2P

DCFR

d. $\lg s$.

ENIAC

HTLM

ICP-Brasil

ISP

SMS

TCP/IP

UNCITRAL

WWW
Código Civil brasileiro (Lei 10.406, de 10 de janeiro de 2002)

Código de Defesa do Consumidor (Lei 8.078, de 11 de setembro de

Contratos business to business

Contratos business to consumer

Contratos Business to public agency

Contratos consumer to public agency

Contratos consumer to consumer

Contratos person to person ou peer to peer

Draft Common Frame of Reference

decreto legislativo (italiano)

Electronic Numerical Integrator and Calculator

Hyper-Text Markup Language

Infraestruturas de Chaves Públicas Brasileiras

Internet Service Provider

Short Message Service

Transmission Control Protocol/ Internet Protocol

United Nations Comission on International Trade Law

World Wide Web 


\section{SUMÁRIO}

INTRODUÇÃ

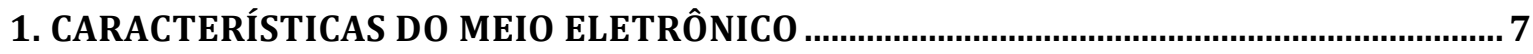

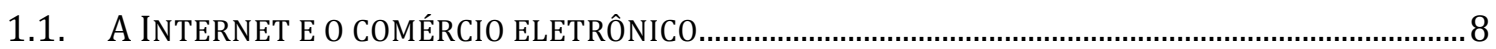

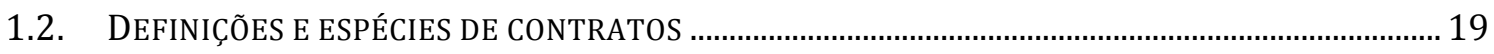

2. O CONTRATO

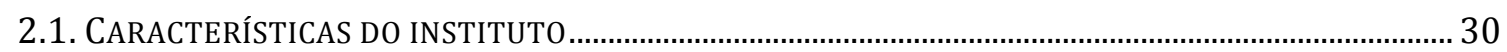

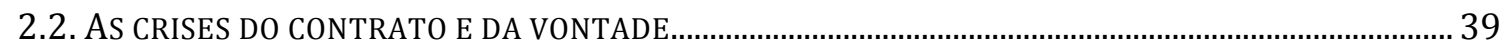

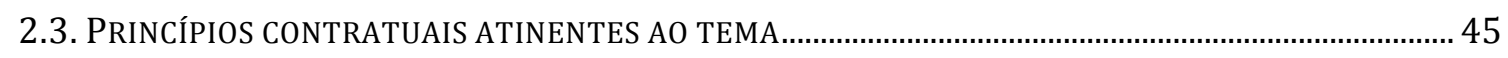

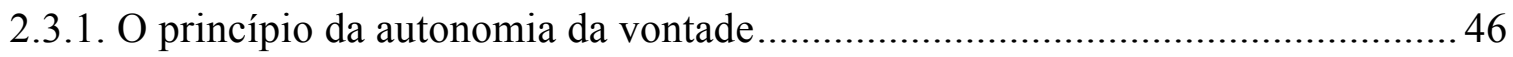

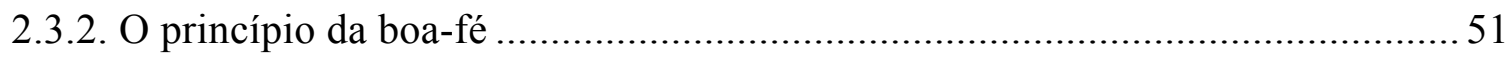

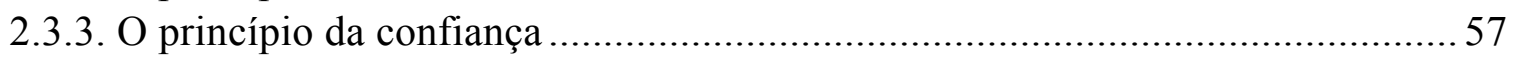

3. A MANIFESTAÇÃO DA VONTADE NA FORMAÇÃO DOS CONTRATOS ELETRÔNICOS 65

3.1. A IMPORTÂNCIA DA DECLARAÇÃO DE VONTADE DAS PARTES E A TEORIA DA APARÊNCIA .................. 67

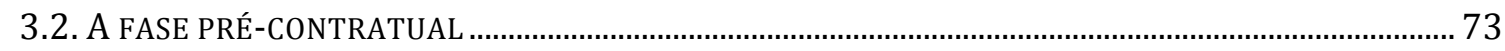

3.3. A FORMAÇÃO MEDIANTE A TROCA DE PROPOSTA E ACEITAÇÃO …………………….......................... 79

3.3.1. Oferta ao público, convite a ofertar e caráter vinculante da publicidade ........... 83

3.4. A FORMAÇÃO DO CONTRATO MEDIANTE O SILÊNCIO ………………………………............................ 89

3.5. A FORMAÇÃO DO CONTRATO MEDIANTE O COMPORTAMENTO CONCLUDENTE E UM CLIQUE.......... 94

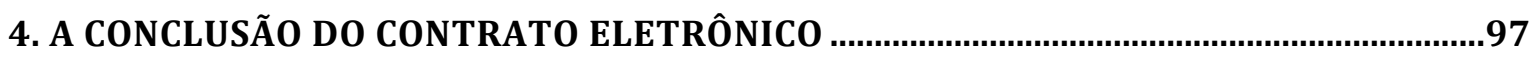

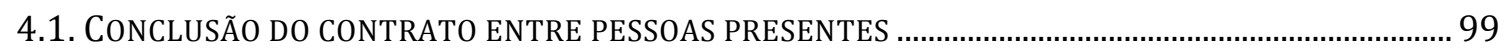

4.2. CONCLUSÃO DO CONTRATO ENTRE PESSOAS AUSENTES ..............................................................101

4.2.1. As teorias da declaração, expedição, conhecimento e recepção ....................... 102

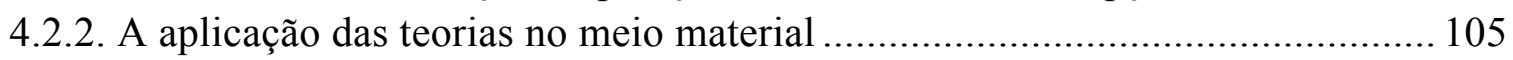

4.2.3. A aplicação das teorias no meio eletrônico ..................................................... 115

4.2.3.1. Peculiaridades dos contratos concluídos mediante cliques e por e-mail ....... 115

4.2.3.2. O embate entre as teorias da expedição e da recepção.................................. 122

4.2.4. A necessidade de utilização da assinatura eletrônica..................................... 131

4.3. O DIREITO DE ARREPENDIMENTO DO CONSUMIDOR ……................................................................137

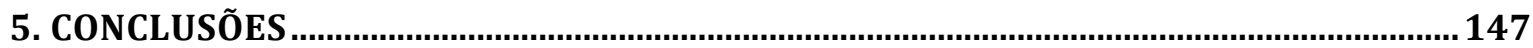

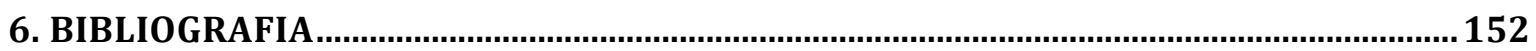




\section{INTRODUÇÃO}

Inserido no fenômeno da globalização de mercados e culturas, o meio eletrônico, representado principalmente pela Internet, quebrou barreiras de espaço e tempo, trazendo diversas facilidades à sociedade.

Esse meio permitiu uma formação contratual diária rápida e prática ${ }^{1}$, havendo o aumento de sua produtividade e rentabilidade, devido à diminuição de tempo e economia de dinheiro, que seriam gastos, por exemplo, com a correspondência entre os contratantes $^{2}$.

Entretanto, devido a algumas de suas peculiaridades, como a ausência de fronteiras entre Estados e a "desumanização"3, os institutos jurídicos já existentes e a sua regulação podem não se aplicar de modo perfeito no mundo imaterial, o que certamente tende a causar grande insegurança jurídica ${ }^{4}$ e vulnerabilidade dos usuários.

O desenvolvimento eletrônico mostra-se progressivamente acelerado ${ }^{5}$. A expansão do número de terminais, como computadores e do uso da Internet, trouxe diversas questões jurídicas, sendo talvez, do campo das relações contratuais, o mais abundante em novidades ${ }^{6}$. Devido à recorrente e crescente utilização dos mecanismos tecnológicos pelo consumidor ${ }^{7}$, mostra-se essencial aprofundar os estudos acerca da

${ }^{1}$ A. P. Gambogi CARvalho, A celebração de contratos via internet segundo os ordenamentos jurídicos alemão e brasileiro, in Revista de Direito do Consumidor, vol. 39, ano 10, 2001, p. 87.

${ }^{2}$ M. H. Diniz, Tratado teórico e prático dos contratos, vol. 5, 6 6 ed., São Paulo, Saraiva, 2006, p. 752. Em poucas palavras, J. OLIVEIRA DE ASCENSÃO demonstra a evolução da internet, que nos últimos anos é um importante local de comércio: "nascida militar, metamorfoseada em científica, massificada a seguir, a Internet foi celeremente transformada num veículo comercial" (Estudos sobre Direito da Internet e da sociedade da informação, Coimbra, Almedina, 2001, p. 18).

${ }^{3}$ G. OPPO, em seu artigo, utiliza a expressão "disumanizzazione" (Disumanizzazione del contrato?, in Rivista di Diritto Civile, vol. 44, n. 5, 1998, pp. 525-533.

${ }^{4}$ Segundo N. LUCCA, a maioria das pessoas ainda sente medo de fazer suas compras por intermédio da rede eletrônica. (Direito e Internet: aspectos jurídicos relevantes I, $2^{\mathrm{a}}$ ed., São Paulo, Quartier Latin, p.70).

${ }^{5}$ F. Galgano, Prefazione, in V. Ricciuto - N. ZORZI (org.), Il contratto telematico, Padova, Cedam, 2002, p. XIII.

${ }^{6}$ A. TAVARES Rosa MARCACINI, Direito e informática: uma abordagem jurídica sobre criptografia, Rio de Janeiro, Forense, 2002, p. 14.

7 "A busca sistemática de ganhos de produtividade por meio de várias formas de uso de aparelhos eletrônicos, computadores e redes de comunicação de dados aos poucos foi tomando conta do conjunto da atividade econômica. Essa tendência continua em nossos dias", evidenciada pelo acesso diário ao ciberespaço (P. LÉvy, Cibercultura, Tradução de C. Irineu da Costa, $3^{\text {a }}$ ed., São Paulo, Editora 34, 2010 , p. $31)$. 
adaptação de institutos tradicionais ao meio eletrônico ${ }^{8}$. E já é possível evidenciar que, para a perfeita concretização do contrato, não basta um simples encaixe do instituto contratual ao novo ambiente ${ }^{9}$.

Surgiram dúvidas quanto a que fonte de direito aplicar a determinadas situações ${ }^{10}$, como as leis, os costumes ou a jurisprudência (que muitas vezes pode até não existir); também passou-se a questionar qual lei dentre as nacional, comunitária e internacional é cabível no regulamento das relações jurídicas ${ }^{11}$; e até se o novo meio precisa de regulamentação ou se deve ser guiado pela autorregulamentação.

No tocante a esse último questionamento, por não ser objeto da presente pesquisa, ousa-se apenas ressaltar que durante toda a exposição, seguir-se-á a opinião que parece a mais apropriada: favorável à regulamentação do meio eletrônico. Segundo G. AlPA, é um erro crer que a globalização e a Internet, pela impossibilidade de sujeitarem-se a fronteiras e, portanto, aos ordenamentos nacionais, são fenômenos não juridificáveis ou que sua regulamentação é inoportuna ${ }^{12}$.

Em um primeiro momento, pensou-se que a simples transposição das normas existentes no mundo material para o mundo real seria sufuciente para evitar a insegurança jurídica, no entanto, posteriormente, percebeu-se a insuficiência de tais normas, que passam cada vez mais a serem utilizadas em um aspecto subsidiário, à medida que normas reguladoras do meio eletrônico são criadas.

Além do campo de mera aplicação legislativa, questões dogmáticas e metodológicas surgiram acerca da formação do contrato. A não imposição de fronteiras

\footnotetext{
${ }^{8}$ De acordo com A. RAWLS, o tema é muito atual, e é importante que se construa uma doutrina que antecipe e acomode desenvolvimentos futuros para que o direito não fique com muitas lacunas em relação à realidade tecnológica (Contract formation in an internet age, in The Columbia Science and technology law review, vol. X, 2009, p. 203, disponível [on-line] in: http://stlr.org/download/volumes/volume10/Rawls.pdf).

${ }^{9}$ Diante da influência do meio eletrônico, o contrato submeteu-se a diversas alterações, fazendo com que os juristas se perguntassem até se ainda seria adequado o uso dos conceitos tradicionais. Cf. F. GAlgano, Prefazione cit. (nota 5 supra), p. XV.

${ }^{10} \mathrm{C}$. CAMARDI, Contratto e rapporto nelle reti telematiche. Un nuovo modelo di scambio, in $\mathrm{V}$. RICCIUTO - N. ZORZI (org.), Il contratto telematico, Padova, Cedam, 2002, p. 3.

${ }^{11} \mathrm{G}$. ALPA afirma que, no tocante aos contratos eletrônicos, a grande problemática dos juristas diz respeito à dúvida de que fonte normativa aplicar. É discutido se esta deve ser nacional, internacional ou até "planetária". Quanto ao enfoque global, como o comércio pode ser realizado entre países que não ratificaram tratados em comum, o consumidor, por exemplo, acaba ficando muito vulnerável e ainda mais exposto ao abuso do fornecedor (Corso di diritto contrattuale, Padova, Cedam, 2006, pp. 410-411).

${ }^{12}$ G. AlPA, Corso di diritto contrattuale cit. (nota 11 supra), pp. 409, 410. Também segue a mesma opinião L. Lessig. De acordo com esse autor, a Internet, que se caracteriza pela anarquia, está, com o passar do tempo, transformando-se em uma rede controlada. A intervenção no "cyberspace" protege as vontades individuais e ao mesmo tempo os valores sociais (Code version 2.0, New York, Basic Books, 2006, pp. 3-6).
} 
e a instantaneidade das operações realizadas na Internet questionaram, principalmente, em qual momento e local é mais apropriada a conclusão contratual eletrônica.

Dentre os possíveis ramos questionados da formação contratual, o tema da presente dissertação de mestrado volta-se à busca da resposta de qual seria o momento mais adequado de conclusão do contrato eletrônico entre ausentes, mais especificamente aquele inserido no âmbito consumeirista.

Os sistemas jurídicos e doutrinas de diferentes países divergem acerca da precisão do exato instante de conclusão do contrato entre ausentes, adotando diferentes teorias, precipuamente as da recepção e da expedição da aceitação. Assim, não há concordância, nem unanimidade doutrinária ou legal a respeito da questão principal, objeto da pesquisa, o que mostra a relevância de seu estudo, inclusive no meio material.

No entanto, foi escolhido o meio eletrônico por duas razões. A primeira por se entender que, caso escolhido o meio tradicional para o estudo do tema, seria necessário compreender de maneira muito detalhada o sistema jurídico de cada país citado, para assim poder apontar o melhor momento de conclusão contratual. E como a aplicação do tema no meio material possui a delimitação de fronteiras, o tema poderia perder a sua importância prática. Isso porque cada nação pode adotar a teoria que mais servir ao seu ordenamento, não havendo, talvez, contribuição algum caso se atinja a conclusão de que teoria diversa seria mais adequada. Assim, cair-se-ia em uma discussão apenas legislativa e comparativa entre os países, sem o rendimento de possíveis frutos.

Como o ciberespaço é desterritorializado, rende maiores discussões e contribuições à análise da formação contratual nele inserida. Nele existe a possibilidade da uniformização legislativa, adotando-se a teoria mais oportuna, em contraposição aos direitos nacionais, que podem, soberanamente, escolher o que lhe soar mais plausível dentro de suas fronteiras delimitadas.

De qualquer forma, procurou-se discutir o tema em um âmbito mais nacional, justamente porque, como ainda não há uma solução, evitou-se perder o enfoque no estudo de diversos sistemas sem a contribuição do brasileiro. No entanto, isso não significa que não haverá a comparação com outros ordenamentos e doutrinas. Isso, na realidade, pode ocorrer por se tratar o tema estudado de âmbito internacinal, sendo que soluções estrangeiras também são pauta de análise, com o cuidado de não misturar os ordenamentos jurídicos, mas sim ponderar se é possível obter uma solução uniforme 
para ser aplicada ao meio eletrônico, ou se é o caso de apenas manter o que é aplicado em cada direito material.

Por último, como o meio imaterial é muito inseguro, devido às suas peculiaridades, mostra-se ainda mais relevante precisar o momento de conclusão contratual.

Também, escolheu-se focar o estudo nos contratos eletrônicos de consumo, pois são muito recorrentes, podendo ocorrer, atualmente, inclusive por meio de aplicativos como o WhatsApp. A exigência, nos contratos entre ausentes, é de uma maior proteção do consumidor quando comparada com a que normalmente já ocorre. Em primeiro lugar, ressalta-se que, nos contratos entre presentes, o consumidor tem, geralmente, a oportunidade de maturação de sua decisão, podendo examinar diretamente o bem objeto da prestação contratual e fazer perguntas ao vendedor. Já no caso das operações concluídas entre ausentes, tanto no meio material quanto imaterial, não existe a possibilidade de exame direto da coisa, e, em regra, não há um diálogo com o vendedor ${ }^{13}$. E nos contratos eletrônicos, além de a maioria ser entre ausentes, acrescenta-se o fato de o consumidor estar ainda mais desprotegido, sujeito a um marketing agressivo, fazendo com que se encontre em uma situação muito vulnerável, por ser induzido a praticamente irresistíveis comportamentos ${ }^{14}$.

Para fins de rigor científico, julgou-se mais adequado apresentar, em primeiro lugar, um panorama que propiciasse subsídios necessários ao desenvolvimento da resposta ao questionamento principal.

Assim, dividiu-se a exposição do tema de maneira que fossem inicialmente contemplados elementos do meio eletrônico, evidenciando suas peculiaridades e seu contexto histórico. Necessária também a exposição da evolução do instituto contratual no meio material, para, assim, posteriormente, ser possível adentrar mais no objeto da pesquisa, com o exame dos meios de manifestação de vontade na formação do contrato eletrônico e a delimitação da natureza jurídica da publicidade na Internet, se seria apenas um convite a ofertar ou já uma oferta ao público, portanto, vinculante.

677.

${ }^{13}$ A. Torrente - P. SChlesinger, Manuale di diritto privato, $21^{\mathrm{a}}$ ed., Milano, Giuffrè, 2013, p.

${ }^{14}$ N. DE LUCCA, A proteção dos consumidores no âmbito da internet, in C. R. PEREIRA DE LIMA, L. N. B. Telles Nunes (org.), Estudos avançados de direito digital, Rio de Janeiro, Elsevier, 2014, p. 98. 
Com o embasamento de tudo discutido, viável a análise do momento de perfeição do acordo nos contratos eletrônicos de consumo entre ausentes, inclusive os mais atuais, concluídos por meio do uso de aplicativos como o WhatsApp. Primeiro, ressaltaram-se as teorias possivelmente aplicadas no meio material, para depois aprofundar seu encaixe no meio imaterial, com o cotejo de elementos que podem ou não influenciar a sua aplicação, como o fato de os contratos eletrônicos serem substancialmente instantâneos, a queda de conexão das partes à rede durante a formação do contrato, a confirmação de recebimento da aceitação do consumidor, dentre outros. Confrontando tais peculiaridades com a aplicação de cada teoria, mostrou-se possível uma sugestão conclusiva.

Ademais, ligados à conclusão contratual, estão a análise do uso da assinatura eletrônica e de sua necessidade, bem como do direito de arrependimento ao consumidor, que, atualmente, é obrigatório em todos os contratos realizados mediante o uso da Internet, de acordo com o direito brasileiro, o que será mais bem explicado no final do texto.

Por fim, cabe, no momento, mencionar os mais relevantes porquês da escolha do tema. A precisão do momento em que um contrato se conclui leva consigo muitas questões de âmbito prático.

Ressalta-se, em primeiro lugar, a determinação do preço, podendo ser acordado que será fixado no instante de conclusão do contrato. Destarte, se, por exemplo, for definido que o contrato se formará quando da expedição da aceitação do consumidor, o preço pode ser um, mas, caso o contrato se torne perfeito quando da recepção da aceitação pelo fornecedor, o preço pode ser outro, menor, ou maior, o que causa imensa insegurança a ambas as partes.

E. GiAntURCo dispõe mais quatro questões relevantes que justificam a exata determinação do momento de conclusão do contrato: precisar o juiz competente, a lei reguladora, o direito de revogar a proposta sem que isso acarrete ônus algum ao proponente e a responsabilidade por caso fortuito ${ }^{15}$.

\footnotetext{
${ }^{15}$ E. GianturCo, Diritto delle obbligazioni, Napoli, Luigi Pierro, 1894, p. 99.
} 
Acrescenta-se também, enfim, a determinação da contagem de prazos, por exemplo, para que o consumidor possa exercer seu direito de arrependimento ${ }^{16}$.

${ }^{16}$ No Brasil, o consumidor, de acordo com o artigo 49 do Código de Defesa do Consumidor, tem direito a arrepender-se em sete dias, sem nenhuma motivação. 


\section{CONCLUSÕES}

No momento, de maneira sintética e pontual, cabe retomar o panorama realizado e individualizar as conclusões e resultados obtidos.

O estudo que resultou na escrita da presente dissertação de mestrado teve como objeto um assunto que mostra embates doutrinários tanto no meio material, quanto no imaterial. Escolheu-se trabalhar com o último, devido às suas diversas peculiaridades, principalmente no tocante à relativização do tempo e do espaço, com a consequente dificuldade da transposição das regras tradicionais aplicadas à formação contratual aos contratos eletrônicos.

Para fins de precisão terminológica, ressaltou-se que a expressão "conclusão", diferente de "formação", refere-se apenas ao momento em que o contrato se torna perfeito, isto é, em que ocorre o encontro de vontade das partes.

Determinar o momento exato de conclusão do contrato eletrônico mostra-se muito relevante, pois pode influenciar na fixação do preço do produto, do foro competente para o julgamento de eventual demanda, da lei reguladora do contrato, do dies a quo do prazo para o exercício do direito de arrependimento do consumidor, e, principalmente, na extinção do direito de revogar a proposta sem que isso acarrete ônus algum ao proponente ou a sua responsabilidade por caso fortuito ou força maior.

Primeiramente, com base no princípio da não discriminação do meio digital, partiu-se da premissa de haver a equivalência entre documentos e comunicações eletrônicos e aqueles advindos do meio material, com a devida adaptação dos institutos jurídicos.

Assim, apesar de o contrato ter sido questionado quando do nascimento do contrato de adesão e, depois, ainda mais em face das peculiaridades do meio eletrônico, entendeu-se que suas etapas de formação merecem apenas uma amoldagem, não sendo necessária a criação de um novo instituto para regular os negócios "sem acordo" e praticamente em silêncio travados na rede mundial de computadores.

Ademais, apesar de a Internet não possuir fronteiras ou materialidade, também se considerou que a acomodação do emprego dos institutos deve ser regulada, ou ao menos as regras já existentes devem ser aplicadas de maneira uniforme. Não pode o novo meio, portanto, basear-se apenas na autorregulamentação, principalmente para não 
haver abusos do fornecedor, que pode se aproveitar da grande vulnerabilidade sofrida pelo consumidor, que sofre grande impacto do marketing no meio eletrônico.

Também, como não se extinguiu o instituto contratual, não há motivos para a não adoção dos princípios clássicos do direito contratual no âmbito dos contratos eletrônicos. Foram estudados os de maior importância para o tema da presente pesquisa: princípios da autonomia da vontade, do pacta sunt servanda, da boa-fé e da confiança.

O primeiro princípio possui restrições, como a lei, a boa-fé e duas limitações de ordem geral: a ordem pública e os bons costumes. No entanto, pelo fato de essas duas ocuparem uma zona cinzenta de delimitação, dependente da cultura de cada época e cada população, existe grande dificuldade de conceituá-las e, consequentemente, de aplicá-las ao meio digital. Viu-se que devido à já mencionada extrema vulnerabilidade do consumidor no e-commerce, é necessário que existam ainda mais restrições ao princípio em análise, o que já vem ocorrendo com a imposição de cláusulas coercitivas, por exemplo, no direito brasileiro, pelo Decreto de $n^{\circ} 7.962$ de 15 de março de 2013.

No tocante ao pacta sunt servanda, sublinhou-se que, apesar de ser necessária à segurança jurídica das relações a aplicação de tal princípio, existe como exceção a ele, além do caso fortuito e da força maior, o direito de arrependimento do consumidor, que é obrigatório nos contratos eletrônicos.

Já no âmbito da boa-fé, percebeu-se sua grande importância nos contratos eletrônicos, principalmente o seu dever anexo de informação e o seu derivado princípio da confiança.

Viu-se que esse último princípio está sendo muito relevante à proteção do consumidor, que deve ter preservada a sua legítima expectativa na formação contratual. Nesse sentido, pode ser aplicada a teoria da aparência, mas com a ressalva de que seu uso é excepcional, por proteger a crença legítima do receptor da mensagem apenas quando é comprovada a sua dificuldade de verificar a situação por meio de uma conduta diligente.

Partindo para uma análise mais específica da formação contratual, considerou-se que as tratativas, convites e pré-contratos não são elementos próprios da formação do contrato, apesar de serem atividades que podem se desenvolver em prol do atingimento do acordo, influenciando em sua formação. 
O cume da formação do contrato é a sua conclusão, que pode ser visualizada no instante do acordo, realizado, principalmente, por meio da troca entre a proposta e a aceitação das partes.

No que diz respeito à oferta, discutiu-se principalmente as diferenças entre a oferta ao público e o convite a ofertar, chegando à conclusão de que a declaração de vontade do fornecedor mostra-se como uma oferta, portanto, vinculante, nos casos em que é possível evidenciar precisamente os requisitos essenciais à formação do contrato.

Dessa forma, a aposição de fotos de mercadorias em uma página de suporte eletrônico e a publicidade, assunto, por sua vez, mais polêmico, têm a natureza jurídica de oferta e não de convite a ofertar. Assim, deve o fornecedor manter o preço e também a disponibilidade do produto, não precisando esta ficar subordinada à disponibilidade no estoque, pois, pelo fato de o contrato eletrônico ser travado em tempo real, é possível haver a programação das vendas de maneira que o anúncio só seja feito até o esgotamento do produto.

Já no que toca à aceitação, considerou-se importante ressaltar outras formas de manifestação da vontade do aceitante, que não seja com a declaração expressa de vontade, dando destaque ao silêncio e ao comportamento concludente.

O contrato formado mediante o comportamento concludente do aceitante conclui-se no momento em que há o início da execução, devendo ele avisar o proponente sobre o início da execução. Afirma-se que nos click-wrap agreements há a formação pelo comportamento do aceitante, porque a manifestação de vontade do consumidor ocorre por meio do seu ato de clicar em um ícone, o que indica que está aderindo às cláusulas formuladas pelo fornecedor e não representa uma declaração expressa de vontade propriamente dita.

No entanto, apesar da afirmativa acima, o contrato eletrônico mediante cliques não é concluído no momento da conduta do consumidor ao clicar no ícone eu aceito ou semelhante, isso porque o clique do consumidor reflete, na realidade, a sua própria aceitação, e não propriamente o início da execução do contrato. O consumidor não necessita, posteriormente ao seu clique, avisar ao proponente que realizou uma ação com vistas à conclusão contratual, e caso se tratasse de um usual contrato com a conclusão por meio de um comportamento concludente, isso teria de ser feito. 
No caso da conclusão dos contratos entre presentes, formam-se pela aceitação imediata da oferta por parte do destinatário, de maneira que é preciso haver a instantaneidade da percepção do proponente de que houve a aceitação do consumidor. Citam-se como exemplos os contratos concluídos em chats virtuais.

No caso dos contratos entre ausentes, constatou-se que não há uma uniformidade doutrinária ou prática quanto ao momento exato de sua conclusão, nem no meio material, nem no imaterial. Para determiná-lo são usadas quatro teorias: do conhecimento, da declaração propriamente dita, da expedição e da recepção.

Após a comparação entre sistemas jurídicos e a ponderação de pontos negativos e positivos principalmente entre a teoria da recepção e da expedição (que, certamente, são as mais adotadas), atingiu-se a conclusão de que a teoria da recepção seria a que melhor se encaixaria ao meio eletrônico.

No meio material, o Brasil adota a teoria da expedição, conforme artigo 434, CC, com mitigações. Mas, apesar de a teoria da expedição ter a vantagem de não precisar apurar o momento da chegada da resposta no destinatário, percebeu-se uma tendência atual de crítica a ela, por fazer sentido apenas em uma época em que a comunicação era muito lenta. Isso porque antigamente resolvia o problema da insegurança do aceitante em confiar que sua manifestação de vontade proferida em uma carta chegaria uma dia ao proponente e formaria o contrato. No entanto, essa insegurança não mais existe diante das inovações tecnológicas, caindo por terra a importância da "mailbox rule".

Ademais, além de outros argumentos expostos no presente trabalho, a teoria da expedição poderia gerar muita insegurança, principalmente aos consumidores, que teriam menos dias para desistir do contrato, caso o prazo referente ao seu direito de arrependimento começasse a contar do envio da aceitação.

Ainda há argumentos e posições contrárias, porém nota-se uma predisposição atual ao seguimento da teoria da recepção, por exemplo, os Princípios Unidroit e o CISG seguem-na expressamente. Inclusive no direito nacional, cita-se o enunciado 173 do Conselho de Justiça Federal, aprovado na Terceira Jornada de Direito Civil.

Adotando-se a teoria da recepção, nos contratos mediante a troca de e-mails, sua conclusão seria efetivada no instante de armazenamento da mensagem, quando se possibilita a sua visualização pelo destinatário. Deve-se fazer a ressalva de que se o $e$ mail é usado para fins particulares e o aceitante não envia sua declaração em horário 
comercial, o contrato só se conclui no dia seguinte; no entanto, caso se trate de um contrato de consumo, pode ser formado inclusive fora do horário comercial, o que ocorre também no caso dos click-wrap agreements. Nesses, após o clique do consumidor no campo "eu aceito" ou similiar, e a consequente recepção dessa aceitação pelo fornecedor, estará perfeito o contrato.

Além de tentar sugerir que a recepção da aceitação pelo proponente seria o melhor momento para a conclusão dos contratos eletrônicos de consumo, inclusive mediante aplicativos como o WhatsApp, foi imprescindível analisar se certas peculiaridades do meio eletrônico influenciariam na determinação de tal momento.

Resumidamente, viu-se a natureza do direito de arrependimento do consumidor, bem como ato de reserva do produto no carrinho de compras; do recebimento, pelo proponente, da aprovação do pagamento feito pelo consumidor; e do envio, pelo proponente, de um aviso de recebimento da aceitação.

Concluiu-se que nenhum desses elementos influencia no momento de conclusão do contrato. A espera, pelo proponente, do recebimento da aprovação do pagamento feito pelo consumidor não posterga a conclusão do contrato, representando apenas uma condição suspensiva do contrato. O direito de o consumidor retratar-se, por sua vez, pode ser considerado uma condição resolutiva, com certas restrições. A obrigatoriedade de o proponente enviar o aviso de recebimento da aceitação é um mero dever anexo, para garantir a segurança jurídica nos relações jurídicas eletrônicas, também não diferindo o momento de conclusão do contrato eletrônico. E, por último, a reserva no carrinho de compras faz parte apenas de uma fase pré-contratual, bem como a eventual aposição da assinatura eletrônica, que não é necessária à formação do contrato, mas é recomendável para garantir maior segurança do consumidor, ao menos a assinatura não qualificada, que se evidencia, por exemplo, pela obrigatoriedade da aposição de uma senha. 


\section{BIBLIOGRAFIA}

Afonso da Silva, Virgílio, Direitos fundamentais, $2^{\mathrm{a}}$ ed., São Paulo, Malheiros, 2010 .

AlPA, Guido, Manuale di diritto privato, 8 ed., Padova, Cedam, 2013. , Corso di diritto contrattuale, Padova, Cedam, 2006.

, Lineamenti di diritto contrattuale, in ALPA, Guido et al., Diritto privato comparato - istituti e problemi, $4^{\mathrm{a}}$ ed., Bari, Laterza, 2012. , L'armonizzazione del diritto contrattuale e il progetto do codice civile europeo, in La nuova giurisprudenza civile comentata, anno XIX, Parte Seconda, Padova, Cedam, 2003. , I contratti di utilizazione del computer, Milano, Giuffrè, 1984.

AlPA, Guido et al., Il contratto in generale II, Torini, G. Giappichelli, 2000.

Antunes Varela, João de Matos, Das obrigações em geral, vol. I, $10^{\mathrm{a}}$ ed., Coimbra, Almedina, 2000.

AsCEnsão, José Oliveira de, Estudos sobre Direito da Internet e da sociedade da informação, Coimbra, Almedina, 2001.

, Contratação em rede informática no Brasil, in Revista TRF - $3^{a}$ Região, vol. 78, jul/ago 2006, p. 59,60.

AZZARI, Federico, La conclusione dei contratti telematiche nel diritto privato europeo, in I Contratti, 2010, p. 301-315.

Barbagalo, Erica Brandini, Contratos eletrônicos, São Paulo, Saraiva, 2001.

Bensoussan, Alain, L'informatique et le droit , vol. II, Paris, Hermes, 1994.

BetTI, Emilio, Teoria generale del negozio giuridico, $2^{\mathrm{a}}$ ed., Napoli, Edizione Scientifiche Italiane, 1994.

Borruso, Renato, Computer e diritto, v. 1, Milano: Giuffrè, 1988.

Bravo, Fabio, Le trattative nei contratto telematici, in I Contratti: Rivista di dottrina e giurisprudenza, n. 7, ano XI, 2003, pp. 739-749.

BrUtTi, Massimo, Dal contratto al negozio giuridico, Torino, G. Giappichelli, 2013.

CAlasso, Francesco, Il negozio giuridico: lezioni di storia del diritto italiano, $2^{\mathrm{a}}$ ed., Milano, Giuffrè, 1968. 
CAMARDI, Carmela, Contratto e rapporto nelle reti telematiche. Un nuovo modelo di scambio, in V. RicciUto e N. ZorZI, Il contratto telematico, Padova, Cedam, 2002.

CARDilli, Riccardo, Bona fides tra storia e sistema, Torino, G. Giappichelli, 2004

Carvalho Santos, J. M., Código civil brasileiro interpretado, v. 15, $2^{\mathrm{a}}$ ed., Rio de Janeiro, Freitas Bastos, 1937.

Cassano, Giuseppe - Cimino, Iacopo Pietro (org.), Diritto dell'Internet e delle nuove tecnologie telematiche, Padova, Cedam, 2009.

Cavalieri Filho, Sergio, Programa de direito do consumidor, São Paulo, Atlas, 2008 .

CHEn, Chung-Wu, Apparence et représentation en droit positif français, in Bibliothèque de droit privé, Tome 340, Paris, L.G.D.J., 2000.

Chirelstein, Marvin A., Concepts and case analysis in the law of contracts, $7^{\mathrm{a}}$ ed., Minnesota, Foundation Press, 2013.

Clive, Eric; Von Bar, Christian (org.). Principles, definitions and model rules of European Private Law: Draft Common Frame of Reference (DCFR), vols. I-II, Munique, Sellier, European Law Publishers, 2009.

CorrêA, Gustavo Testa, Aspectos jurídicos da Internet, São Paulo, Saraiva, 2000

D’Angelo, Andrea, Accordo e procedimenti di formazione, in V. Roppo, Tratatto del contratto I - Formazione, Milano, Giuffrè, 2006, pp. 1-174.

Danis-Fatôme, Anne, Apparence et contrat, in Bibliothèque de droit privé, Tome 414, Paris, L. G.D.J., 2004.

DE LuCCA, Newton, Aspectos jurídicos da contratação informática e telemática, São Paulo, Saraiva, 2003. , A proteção dos consumidores no âmbito da internet, in PEREIRA DE Lima, Cíntia Rosa - Telles Nunes, Lydia Neves Bastos (org.), Estudos avançados de direito digital, Rio de Janeiro, Elsevier, 2014, pp. 91-103.

De LuCCA, Newton - Simão FILHO, Adalberto (org.), Direito e Internet: aspectos jurídicos relevantes I e II, São Paulo, Quartier Latin, 2005-2008.

De-Mattia, Fábio Maria, Aparência de representação, São Paulo, Gaetano 
Dibenedetto, 1999.

Dholakia, Nikhilesh, Fritz, Wolfgang, Dholakia, Ruby Roy e Mundorf, Norbert, Global e-commerce and online marketing, Quorum Books, Westport (CT), 2002.

DINIZ, Maria Helena, Tratado teórico e prático dos contratos, vol. 5, 6a ed., São Paulo, Saraiva, 2006.

Domínguez Olmos, Javier, Deberes de protección, 'aun frente a terceros', en la dogmatica alemana, in Revista de Derecho Privado, Colômbia, enero-junio 2011, pp. 301-346.

EISLER, Beth A., Default rules for contract formation by promise and the need for revision of the mailbox rule, in Kentucky Law Journal, vol. 79, 1991, pp. 557-568.

Elias, Paulo Sá, Contratos eletrônicos e a formação do vínculo, São Paulo, Lex Editora, 2008.

EISEnberg, Melvin A., The theory of contracts, in Benson, Peter, The theory of contract law - new essays, New York, Cambridge University Press, 2001.

ENNECCERUS, Ludwig, Lehrbuch der bürgerlichen Rechts - Einführung und allgemeiner, Teil I, Marburg, N. G. Elwert, 1928.

Escorel, Clarisse - Leonardos, Gabriel F., Brazil, in Kinsella, N. Stephan SimPSON, Andrew F., Online contract formation, $1^{\text {a }}$ ed., New York, Oceana Publications, 2004, in Kinsella, N. Stephan e Simpson, Andrew F., Online contract formation, $1^{\text {a }}$ ed., New York, Oceana Publications, 2004, pp. 21-30.

Farnsworth, Edward Allan, Contracts, $2^{\mathrm{a}}$ ed., New York, Aspen Publisher, 1990.

FASCIANO, Paul, Internet electronic mail: a last bastion for the mailbox rule, in Hofstra Law Review, vol. 25, 1997, pp. 971-1003, disponível [on-line] in: http://scholarlycommons.law.hofstra.edu/hlr/vol25/iss3/8/

FAVA, Pasquale, Il contratto nella teoria generale del diritto, in FAVA, Pasquale (org.), Il contratto, Milano, Giuffrè, 2012, pp. 4-53.

FERRARI, Filippo, La formazione del contratto, in F. GALGAno (org.), Atlante di diritto privato comparato, $5^{\mathrm{a}}$ ed., Bologna, Zanichelli, 2011. 
FErreIrA, Patrícia Cândido Alves, Princípio da confiança: proteção e tópica jurisprudencial dos contratos de saúde suplementar, in Revista de Direito Civil Contemporâneo, vol. 2, jan-mar 2015, pp. 83-107.

FERRO-LuZZI, Federico, L'imputazione precontrattuale: il preliminare, le trattative, Padova, Cedam, 1999.

Finkelstein, Maria Eugênia Reis, Aspectos jurídicos do comércio eletrônico, Porto Alegre, Síntese, 2004.

Finocchiaro, Giusella, I contratti ad oggeto informatico, Padova, Cedam, 1993.

Fortinguerra, Fabio e DE MAUro, Antonio, La responsabilità precontrattuale, Padova, Cedam, 2002.

Franceschelli, Vincenzo, Premesse generali per uno studio del commercio elettronico, in Franceschelli, Vincenzo (org.), Commercio elettronico, Milano, Giuffrè, 2001, pp. 2-48.

Fraternale, Antonio, I contratti a distanza, Milano, Giuffrè, 2002.

FRAU, Riccardo, La formazione e l'invalidità del contratto telematico, in Ricciuto, Vincenzo (org.), Il contratto telematico e $i$ pagamenti elettronici: l'esperienza italiana e spagnola a confronto, Milano, Giuffrè, 2004, pp. 35-52.

GAlgano, Francesco, Il negozio giuridico in Germania e in Italia, in F. Galgano (org.), Atlante di diritto privato comparato, $5^{\mathrm{a}}$ ed., Bologna, Zanichelli, 2011, pp. 55-60. , Il contratto, $2^{\mathrm{a}}$ ed., Padova, Cedam, 2011. , Commentario breve al codice civile, Piacenza, La Tribuna, 2006. , Prefazione, in Ricciuto, Vincenzo e ZorzI, Nadia (org.), Il contratto telematico, Padova, Cedam, 2002. , Diritto Privato, 6 ${ }^{\mathrm{a}}$ ed., Padova, Cedam, 1990. , Il negozio giuridico, in Trattato di Diritto Civile e Commerciale, Milano, Giuffrè, 1988.

GAMBINO, Alberto Maria, I principi regolativi dei contrats télématiques, in Diritto del commercio internazionale - pratica internazionale e diritto interno, Milano, Giuffrè, 1997, pp. 295-322. 
Gambogi Carvalho, Ana Paula, A celebração de contratos via internet segundo os ordenamentos jurídicos alemão e brasileiro, in Revista de Direito do Consumidor, ano 10, n. 39, julho-setembro 2001, pp. 85-117.

Gastão Paes de Barros LeÃes, Luis, Notas sobre boa-fé e lealdade negocial, in Principi per $i$ contratti commerciali internazionali e il sistema giuridico latinoamericano, Padova, CEDAM, 1996.

Gaudemet, Eugène; Gaudemet, Jean; Debois, Henri, Théorie générale des obligations, Paris, Sirey, 1965.

GAZzonI, Francesco, Manuale di diritto privato, $16^{\mathrm{a}}$ ed., Napoli, Edizioni Schientifiche Italiane, 2013. , Il contratto preliminare, $2^{\mathrm{a}}$ ed., Torino, G. Giappichelli, 2002.

Gianturco, Emanuele, Diritto delle obbligazioni, Napoli, Luigi Pierro, 1894.

GiLmore, Grant, The death of contract, Columbus, Ohio State University Press, 1974.

GiUsti, Alberto e PARADINI, Mauro, Il contratto preliminare, Milano, Giuffrè, 1992.

Gomes, Orlando, Contratos, $25^{\mathrm{a}}$ ed., Rio de Janeiro: Forense, 2002.

Gorla, Gino, El contrato - problemas fundamentales tratados según el método comparativo y casuístico, Barcelona, Bosch, 1959.

GRAF, Cristoph, Der Abschkuss elektronischer Verträge, in Recht der Internationalen Wirtschaft, 48. Jahrgang, n. 3, Heidelberg, Recht und Wirtschaft, 2002, pp. 179-182.

Gramunt Fombuena, Maria Dolores, La formación y la invalidez del contratto electrónico, in RICcIUTO, Vincenzo, Il contratto telematico e i pagamenti elettronici: l'esperienza italiana e spagnola a confronto, Milano, Giuffrè, 2004, pp. 17-34.

IPPOLITO, Fulvio Sarzana di S., I contratti di Internet e del commercio eletronico, Milano, Giuffrè, 2001.

IRTI, Natalino, È vero ma... (Replica a Giorgio Oppo), in Rivista di diritto civile, Cedam, Padova, Anno XLV, 1999, pp. 273-278. , Scambi senza acordo, in Rivista Trimestrale di Diritto e Procedura Civile, v. LII, n. 2, 1998, pp. 347-364.

JHERING, Rudolf Von, Culpa in contrahendo oder Schadenersatz bei nichtigen 
oder nicht zur Perfection gelangtei Vertragen, 1860, trad. port. de P. Mota Pinto, Culpa in Contrahendo ou indemnização em contratos nulos ou não chegados à perfeição, Coimbra, Almedina, 2008.

JunQueIRA DE AzEVEDo, Antônio, Negócio jurídico - existência, validade e eficácia, $4^{\mathrm{a}}$ ed., São Paulo, Saraiva, 2010.

, Novos estudos e pareceres de direito privado, $1^{\text {a }}$ ed., São Paulo,

Saraiva, 2009.

, Natureza jurídica do contrato de consórcio. Classificação dos atos jurídicos quanto ao número de partes e quanto aos efeitos. Os contratos relacionais. A boa-fé nos contratos relacionais. Contratos de duração. Alteração das circunstâncias e onerosidade excessiva. Sinalagma e resolução contratual. Resolução parcial do contrato. Função social do contrato, in Revista dos Tribunais, ano 94, v. 832, fev. 2005, pp. 115-137.

KESSLER, Friedrich, Contracts of adhesion - some thoughts about freedom of contract, 43, Columbia Law Review, 1943, pp. 629-641.

Kessler, Friedrich, Gilmore, Grant, Contracts: cases and materials, $2^{\mathrm{a}}$ ed., Boston, Brown, 1970.

KLEIN, Vinícius, As contratações eletrônicas interempresariais e o princípio da boa-fé objetiva: o caso do EDI, in MARTIns, Guilherme Magalhães (org.), Direito privado e internet, São Paulo, Atlas, 2014, pp. 383-398.

KÜMPEL, Vitor Frederico, A teoria da aparência no novo Código Civil brasileiro: lei 10.406, de 10 de janeiro de 2002, Tese (Doutorado em Direito), Faculdade de Direito, Universidade de São Paulo, São Paulo, 2004. , Teoria da aparência no Código Civil de 2002, São Paulo, Método, 2007.

LEAL, Sheila do Rocio Cercal Santos Leal, Contratos eletrônicos: validade jurídica dos contratos via Internet, São Paulo, Atlas, 2007.

Lessig, Lawrence, Code - version 2.0, New York, Basic Books, 2006. , Code and other laws of cyberspace, New York, Basic Books, 1999.

LÉVy, Pierre, Qu'est-ce que le virtuel?, Paris, La Découverte, 1996, trad. port. de P. Neves, O que é virtual, São Paulo, Editora 34, 1996.

, Cyberculture, Paris, Odile Jacob, 1997, trad. port. de Costa, C. Irineu 
da, Cibercultura, $3^{\mathrm{a}}$ ed, São Paulo, Editora 34, 2010.

Lima Marques, Cláudia (org.), A nova crise do contrato - estudos sobre a nova teoria contratual, São Paulo, Revista dos Tribunais, 2007.

Lima Marques, Cláudia, Confiança no comércio eletrônico e a proteção do consumidor - um estudo dos negócios jurídicos de consumo no comércio eletrônico, São Paulo, Revista dos Tribunais, 2004.

, Contratos no Código de Defesa do Consumidor, $6^{\text {a }}$ ed., São Paulo, Revista dos Tribunais, 2011.

, Comércio eletrônico de consumo internacional: modelos de aplicação da lei mais favorável ao consumidor e do privilégio de foro, in Revista do Advogado, n. 114, AASP, São Paulo, dez. 2011, pp. 33-34.

Lorenzetti, Ricardo L., Comercio Eletrónico, 2001, trad. port. de F. Menke, Comércio Eletrônico, São Paulo, Revista dos Tribunais, 2004.

Losano, Mario G., I grandi sistemi giuridici: introduzione ai diritti europei ed extraeuropei, Torino, Piccola Biblioteca Enaudi, 1978.

LUCIDI, Irina, La recezione del negozio giuridico in altri paesi, in F. GALGANO (org.), Atlante di diritto privato comparato, $5^{\text {a }}$ ed., Bologna, Zanichelli, 2011, pp. 60-67.

Luhmann, Niklas, Confianza, Barcelona, Anthropos Editorial, 2005.

MaCAUlay, Stewart, Non-contratual relations in Business: a preliminary study, American Social Review, n. 1, v. 28, February, 1963.

MARTIns, Guilherme Magalhães, Formação dos contratos eletrônicos de consumo via internet, $2^{\mathrm{a}}$ ed., Rio de Janeiro, Lumen Juris, 2010.

MalfitAno, Daliela, Le trattative e la responsabilità preconttratuale, in $\mathrm{M}$. Bessone (org.), Casi e questioni di diritto privato, v. XXI - Il contratto in generale, Milano, Giuffrè, 2002.

Marcacini, A. T. Rosa, Direito e informática: uma abordagem jurídica sobre criptografia, Rio de Janeiro, Forense, 2002.

Marini, Giovanni, Promessa e affidamento nel diritto dei contratti, Napoli, Jovene, 1995.

Massimo Bianca, Cesare, Diritto Civile III - Il contratto, $2^{\mathrm{a}}$ ed., Milano: Giuffrè, 2000.

Mattos, Analice Castor de, Aspectos relevantes dos contratos de consumo 
eletrônico, Curitiba, Juruá, 2009.

Mckendrick, Ewan, Contract law, 10 a ed., Basingstoke, Palgrave Macmillan, 2013.

Medicus, Dieter, Tratado de las relaciones obligacionales, trad. espanhola de A. Martínez Sarrión, vol. 1, Barcelona, Bosch, 1995.

Menezes Cordeiro, António Manuel da Rocha, Da boa-fé no direito civil, $4^{\mathrm{a}}$ reimp., Coimbra, Almedina, 2011.

, Tratado de direito civil português II - direito das obrigações, t. II, Coimbra, Almedina, 2010.

Menke, Fabiano, Assinatura eletrônica no direito brasileiro, São Paulo, Revista dos Tribunais, 2005.

Messineo, Francesco, Il contratto in genere, vol. I, Milano, Giuffrè, 1973.

MiLls, Karen, Effective formation of contracts by electronic means and dispute resolution in the new e-conomy: still more questions than answers, in KInSELLA, N. Stephan e SimPSON, Andrew F., Online contract formation, $1^{\mathrm{a}}$ ed., New York, Oceana Publications, 2004, pp. 431-443.

Minervini, Enrico, Bartolomucci, Pierfrancesco, La tutela del consumatore telematico, in VAlentino, Daniela (org.), Manuale di diritto dell'informatica, $2^{\mathrm{a}}$ ed., Napoli, Edizione Scientifiche Italiane, 2011, pp. 347-364.

Miranda, C. P. Ubaldino, Comentários ao Código Civil-dos contratos em geral, v. 5, São Paulo, Saraiva, 2013.

Modenesi, Pedro, Contratos eletrônicos de consumo: aspectos doutrinário, legislativo e jurisprudencial, in MARTINS, Guilherme Magalhães (org.), Direito privado e internet, São Paulo, Atlas, 2014, pp. 301-369.

Mota Pinto, Carlos Alberto da, Teoria geral do direito civil, $4^{\mathrm{a}}$ ed., Coimbra, Almedina, 2005.

Neme Villarreal, Martha Lucía, La buena fe en el derecho romano - extensión del deber de actuar conforme a buena fe en materia contratual, Universidad Externado de Colombia, Bogotá, 2010.

NerY JÚNIOR, Nelson, Da proteção contratual, in GrINOver, Ada Pellegrini (org.), Código brasileiro de defesa do consumidor: comentado pelos autores do anteprojeto, $9^{\text {a }}$ ed., Rio de Janeiro, Forense, 2007. 
Nivarra, Lucas, Ricciuto, Vincenzo, Scognamiglio, Claudio, Istituzioni di diritto privato, $6^{\mathrm{a}}$ ed., Torino, Giappichelli, 2011.

Nunes Barbosa, Fernanda, Informação e consumo: a proteção da privacidade do consumidor no mercado contemporâneo da oferta, in MARTINS, Guilherme Magalhães (org.), Direito privado e internet, São Paulo, Atlas, 2014, pp. 233-257.

OliveIRA, Elsa Dias, A proteção dos consumidores nos contratos celebrados através da internet, Coimbra, Almedina, 2002.

Oppo, Giorgio, Disumanizzazione del contrato?, in Rivista di Diritto Civile, n.5, vol. 44, 1998, pp. 525-533.

OwsIA, Parviz, Formation of contract - a comparative study under English, French, Islamic and Iranian Law, London, Grahan \& Trotman, 1993.

PARISI, Francesco, Il contratto concluso mediante computer, Padova, Cedam, 1987.

Pereira, Caio Mário da Silva, Instituições de Direito Civil, vol. III - Contratos, $15^{\mathrm{a}}$ ed., São Paulo, Forense, 2011.

Pereira De Lima, Cíntia Rosa, Validade e obrigatoriedade dos contratos de adesão eletrônicos (Shrink-Wrap e Click-Wrap) e dos termos e condições de uso (Browse-Wrap): um estudo comparado entre Brasil e Canadá, Tese (Doutorado em Direito), Faculdade de Direito, Universidade de São Paulo, São Paulo, 2009.

, Contratos de adesão eletrônicos (shrink-wrap e click-wrap) e os termos de condições de uso (browse-wrap), in PEREIRA DE LIMA, Cíntia Rosa - Telles NunEs, Lydia Neves Bastos (org.), Estudos avançados de direito digital, Rio de Janeiro, Elsevier, 2014, pp. 105-133.

Perlingieri, Giovanni, Il contratto telematico, in VAlentino, Daniela (org.), Manuale di diritto dell'informatica, $2^{\mathrm{a}}$ ed., Napoli, Edizione Scientifiche Italiane, 2011, pp. 266-287.

Perlingieri, Pietro, Manuale di diritto civile, $6^{\mathrm{a}}$ ed., Napoli, Edizione Schientifiche Italiane, 2007.

PinheIro, Patrícia Peck Garrido, Direito digital, $3^{\mathrm{a}}$ ed., São Paulo, Saraiva, 2009.

Pontes de Miranda, Francisco Cavalcanti, Tratado de direito privado - II, III e IV, São Paulo, Revista dos Tribunais, 2012. 
QueIroz De Moraes, Bernardo Bissoto, A noção romana de assinatura, in Revista da Faculdade de Direito - Fundação Armando Álvares Penteado, v. 7, jan-jun 2012, pp. 14-29.

RAMSAY, Iain, Consumer protection in the era of informational capitalism, in Wilhelmsson, Thomas, Tuominem, Salla, Tuomola, Heli, Consumer Law in the information society, The Hague, Kluer Law International, 2001.

RAVAZZONI, Alberto, La formazione del contratto - le regole di comportamento, Milano, Giuffrè, 1974.

RAWLS, Amelia, Contract formation in an internet age, The Columbia Science and technology law review, vol. X, 2009, pp. 200-231, disponível [on-line] in: http://stlr.org/download/volumes/volume10/Rawls.pdf

ReICH, Dietmar O., ScHMITZ, Peter, Einführung in das Bürgerliche Recht, $3^{\mathrm{a}}$ ed., Göttingen, Gabler, 2000.

RICCIUTO, Vincenzo, Formazione progressiva del contratto ed obblighi a contrarre, $2^{\mathrm{a}}$ ed., Torino, UTET, 2006.

Ricciuto, Vincenzo e ZorzI, Nadia (org.), Il contratto telematico, Padova, Cedam, 2002.

RicciUto, Vincenzo, La formazione del contratto telematico e la tutela del consumatore, in RICCIUTO, Vincenzo (org.), Il contratto telematico e $i$ pagamenti elettronici: l'esperienza italiana e spagnola a confronto, Milano, Giuffrè, 2004, pp. 116.

RoDiere, Rene (org.), Harmonisation du droit des affaires dans les pays $d u$ marche commun - La formation du contrat, Paris, Pedone, 1976.

Rodrigues, Silvio, Direito Civil - dos contratos e das declarações unilaterais de vontade, v.3, 30 a ed., São Paulo, Saraiva, 2004.

Rolli, Rita, Antiche e nuove questione sul silenzio come tacita manifestazione di volontà, in Contratto e impresa, n. 1, ano 16, 2000, pp. 207-286.

RopPo, Vincenzo, Il contratto, Giuffrè, Milano, 2001. , Il contratto del duemila, $3^{\mathrm{a}}$ ed. Torino, G. Giappichelli, 2000.

Rossello, Carlo, La nuova disciplina del commercio elettronico. Principi generali e ambito di applicazione, in Diritto del commercio internazionale - pratica internazionale e diritto interno, Milano, Giuffrè, 2004, pp. 43-67. 
, Gli oblighi informativi del prestatore di servizi, in Rossello, Carlo, Finocchiaro, Giusella e Tosi, Emilio (org.), Commercio elettronico, documento informatico e firma digitale, Torino, G. Giappichelli, 2003, pp. 35-60.

Russo, Stefano e ScAVIZZI, Roberto, Manuale di diritto comunitario dell'informatica, Milano, Giuffrè, 2010.

SAMmARCo, Emilio, I contratti dell'informatica, in LiPARI, Nicolò, Rescigno, Pietro (org.), Diritto Civile - Obbligazioni: I contratti, v. III, Milano, Giuffrè, 2009, pp. 775-797.

Santos, Manoel Pereira e Rossi, Mariza Delapieve, Aspectos legais do comércio eletrônico - contratos de adesão, in Revista do direito do consumidor, n. 36, out./dez. 2004, pp. 105-129.

SAVIGNY, Friedrich Carl von, Obligationenrecht, 1853, trad. italiana de Pacchioni, Giovanni, Le obbligazione, v. II, Torino, Unione Tipografico, 1915.

SCHMidT, Jan Peter, La revocación de la oferta en el derecho contractual de los países y en el derecho uniforme, in Roma e America. Diritto Romano Comune - Rivista di diritto dell'integrazione e unificazione del diritto in Europa e in America Latina, vol. 30, Roma, Mucchi, 2010, pp. 135-147.

, Vida e obra de Pontes de Miranda a partir de uma perspectiva alemã - com especial referência à tricotomia 'existência, validade e eficácia do negócio jurídico', in Revista Fórum de Direito Civil, Belo Horizonte, ano 3, n. 5, jan-abril 2014, disponível [on-line] em http://www.direitocontemporaneo.com/wpcontent/uploads/2014/02/SCHMIDT-

VidaeobradePontesdeMirandaapartirdeumaperspectivaalema.pdf .

SCHOUERI, Luís Eduardo (org.), Internet: o direito na era virtual, 2a ed., Rio de Janeiro: Forense, 2001.

Scialoja, Vittorio, Negozi Giuridici, Roma, Foro Italiano, 1933.

SiCA, Salvatore, ZENO-ZENCOVICH, Vincenzo, Legislazione, giurisprudenza e dottrina nel diritto dell'internet, in Il diritto dell'informazione e dell'informatica, anno XXVI, n. 3, Milano, Giuffrè, 2003, pp. 377-389.

Somma, Alessandro, Towards a European private law? The Common Frame of Reference in conflict between EC law and national laws, in MickLITZ, Hans-W., CAFAGGI, Fabrizio (org.), European private law after the Common Frame of Reference, 
Massachusets, Edward Elgar, 2010, pp. 1-23.

Stewart, Concetta, Tian, Yan, History of e-commerce, in KHOSROW-Pour, Mehdi (org.), Encyclopedia of e-commerce, e-government, and mobile commerce, London, 2006, pp. 559-564.

Stiglitz, Rosana M., Contratos celebrados por medio de computadoras, in Stiglitz, Rúben S. Contratos teoría general, vol. I, Buenos Aires, Depalma, 1990.

Stolfi, Giuseppe, Teoria del negozio giuridico, Padova, Cedam, 1961.

TANG, Zheng Sophia, Electronic consumer contracts in the conflict of laws, Oxford and Portland, Hart publishing, 2009.

TeIXeIra, Tarcisio, Comércio eletrônico: conforme o Marco Civil da Internet e a regulamentação do e-commerce no Brasil, São Paulo, Saraiva, 2015.

TOMASETti Junior, Alcides, Execução do contrato preliminar, Tese (Doutorado em Direito), Faculdade de Direito, Universidade de São Paulo, São Paulo, 1982.

TOMASEVICIUS FILHO, Eduardo, Informação assimétrica, custos de transação, princípios da boa-fé, Tese (Doutorado em Direito), Faculdade de Direito, Universidade de São Paulo, São Paulo, 2007.

Torrente, Andrea - Schlesinger, Piero, Manuale di diritto privato, $21^{\mathrm{a}}$ ed., Milano, Giuffrè, 2013.

Tosi, Emilio, Contratazione telematica e conclusione del contratto virtuale, in Rossello, Carlo, Finocchiaro, Giusella e Tosi, Emilio (org.), Commercio elettronico, documento informatico e firma digitale, Torino, G. Giappichelli, 2003, pp. 97-130.

TrabuCCHI, Alberto, Istituzioni di diritto civile, 44a ed., Padova, Cedam, 2009.

Ulhôa Coelho, Fábio, Direitos do consumidor no comércio eletrônico, in Revista do Advogado, São Paulo, Associação dos Advogados de São Paulo, n. 89, 2006.

VAnZella, Rafael Domingos Faiardo, O contrato, de enzo a vincenzo, in Revista Direito GV, v.1, n.2, 2005, pp. 221-227.

VerçosA, Haroldo Malheiros Duclerc, Agente fiduciário do consumidor em compras pela internet: um novo negócio nascido da criatividade mercantil, in Revista de Direito Mercantil, Industrial, Econômico e Financeiro, São Paulo, v. 118, 2000, pp. 88-93.

WATNICK, Valerie J., The electronic formation of contracts and the Common Law 'Mailbox Rule', in Baylor Law Review, New York University, Baruch College, vol. 56, 
n. 175, 2004, pp. 175-2003, disponível [on-line] in http://heinonline.org/HOL/LandingPage?handle=hein.journals/baylr56\&div=11\&id=\&pa $\mathrm{ge}=$

Weitzenbock, Emily M., Good Faith and fair dealing in contracts formed and performed by electronic agentes in Artificial Intelligence and Law, vol. 12, n. 1-2, 2004, Netherlands, Springer, pp. 83-110. Disponível [on-line] em: http://ssrn.com/abstract $=2173223$.

WINDSCHEID, Bernhard, Lehrbuch des Pandektenrechts, v. 1, trad. italiana de C. Fadda e P. E. Bansa, Diritto delle pandette, Torino, Unione Tipografico, 1902.

ZAtTi, Paolo, Diritto Privato - corso istituzionale, $3^{\text {a }}$ ed., Padova, Cedam, 2010.

Zimmermann, Reinhard, The law of obligations: Roman foundation of the civilian tradition, London, Oxford, 1996.

Zimmermann, Reinhard e WhitTAKer, Simon, Good Faith in European Contract Law, Cambridge, Cambridge University Press, 2000.

ZopPINI, Andrea, La conclusione dei contratti su internet, Jei - Juris e Internet, 1999, disponível [on-line] em: http://www.jei.it/approfondimenti/item/342-laconclusione-dei-contratti-su-internet. 